\title{
An inference procedure for behavioural studies combining numerical simulations, statistics and experimental results
}

\author{
JEAN-MARC GUARINI ${ }^{1,2}$, JENNIFER COSTON-GUARINI ${ }^{1,2}$, TIM DEPREZ ${ }^{3}$ AND LAURENT CHAUVAUD ${ }^{2}$ \\ ${ }^{1}$ The Entangled Bank Laboratory, 66650 Banyuls sur Mer, France, ${ }^{2}$ CNRS/UBO - UMR 6539 LEMAR/LIA BeBEST (Benthique \\ Biodiversity, Ecology, Sciences et Technologies), Rue Dumont d'Urville, 2928o Plouzané, France, ${ }^{3}$ Marine Biology Research Group, \\ Krijgslaan 281/S8, B9ooo Gent, Belgium
}

\begin{abstract}
The technical difficulties of performing underwater observation mean that marine ecologists have long relied on behavioural experiments to study reactions of marine organisms. In this article, we examine the underlying complexity of assumptions made in raceway experiments and we propose a statistical inference procedure tailored to this type of experimental protocol. As an example, experiments were performed to test if light of two different intensities affects the proximal behaviour (i.e. direct, local and immediate) of two species of crustaceans, the hermit crab (Pagurus bernhardus), and the green crab (Carcinus maenas). Individuals were collected in the vicinity of the Sven Loven Marine Center in Tjarnö (Sweden). Their movements in raceways were recorded and the statistical distance between the resulting experimental distribution and a simulated null distribution was used to compare their behaviour in two situations: dim (when they were expected to feed) and bright light (when they were expected to shelter). Initial tests indicated no differences of behaviour between dim and bright light for the two species. However, when compared with the reference state (here, a null distribution) the behaviour in dim light deviates significantly from the null distribution suggesting non-random behaviour. Our results suggest that efforts should be made to understand the behaviours of the individuals of these two species to establish a comprehensive reference state as a basis for comparison. This fundamental information should be a prerequisite before implementing experiments testing how potential disturbances affect individual organisms in behavioural ecology.
\end{abstract}

Keywords: Crab individual behaviours, light pollution, microcosms, probability, null distribution

first published online 8 November 2017

\section{INTRDDUCTION}

Behavioural studies are usually motivated by questions about how organisms respond to environmental cues that can be tested experimentally in controlled conditions (Martin \& Bateson, 2007; Davies et al., 2012). However, even if experiments are designed to study 'proximal causation' (Martin \& Bateson, 2007, evoking Tinbergen's Rules), the fundamental ecological questions concern ecological fitness and adaptive strategies (Reynolds, 2014). In this context, one of the persistent challenges remains how experimental results are interpreted, leading to misunderstanding and controversies (Ylonen \& Wolff, 1999; Chapman, 2000). Therefore, in addition to experimental controls and replication, results are often compared with null model outcomes (Vickers \& Schwarzkopf, 2016). Null models represent the free distribution, i.e. the set of behavioural mechanisms seen as pure random processes, as opposed to what organisms can perform if they develop deterministic abilities to optimize certain activities.

In this article, we explore how raceway-type experiments with intertidal invertebrates could benefit from this conceptualization. Raceway experiments are apparently simple experiments used to demonstrate avoidance or attraction

Corresponding author:

J. Coston-Guarini

Email: jm.guarini@entangled-bank-lab.org behaviours. These have been a mainstay of ecological studies for decades (Campbell et al., 2009). We propose a statistical inference procedure tailored to this type of experimental protocol. We elected to test, as an example, reactions to light, because light 'pollution' has been identified as a threat for marine species and biodiversity inhabiting urbanized coastal areas (Navarro-Barranco \& Hughes, 2015). Early studies have started to quantify light pollution, attempting to define precise indicators at different scales of perception (Cinzano et al., 2001; Cinzano \& Falchi, 2014). While experimental studies of light pollution effects on aquatic systems are still scarce, it has been suggested that it can affect vertical migration of daphnia (Moore et al., 2000), biological rhythms via the night-time production of melatonin in perch (Brüning et al., 2015), and phytoplankton production and physiology (Poulin et al., 2013). In addition, light pollution was suggested to modify benthic diversity (NavarroBarranco \& Hughes, 2015), but, despite the fact that benthic invertebrates exhibit a strong circadian behavioural rhythm (Brady, 2013), few studies exist concerning the alteration of individual behaviours of common shore species.

We focused on the behaviours of two crab species, the hermit crab (Pagurus bernhardus), common to the North-east Atlantic coastal waters and the green crab (Carcinus maenas), which has dispersed worldwide (Carlton \& Cohen, 2003). Both species exhibit a protective behaviour to shelter, and a circadian activity pattern, sheltering during 
daylight and predation during night-time (Burrows et al., 1999; Turra \& Denadai, 2003). Both consume a very large range of food resources (Cohen \& Carlton, 1995), but adult individuals of Carcinus maenas are mainly predators (Pihl, 1985) with a strong preference for mussels (McGaw \& Penney, 2014), while adults of hermit crabs are mainly generalists and opportunistic scavengers (Nickell \& Moore, 1992) with the olfactory ability to detect what is available (Tran, 2015). Both species have intra- and interspecific aggressive behaviours which can blur the quantification of individual foraging behaviours in their natural environment (Ramsay et al. 1997; Quinn et al., 2012; Chakravarti \& Cotton, 2014).

\section{MATERIALS AND METHDDS}

\section{Concepts}

The experiment was conceived to test the role of an environmental factor (here artificial light intensity) on two antagonistic behaviours, in our case, the instinct to shelter and the need to feed. Conceptualized in the simplest manner, individuals can explore a defined space in which at least one place offers shelter and another distinct one offers a food source
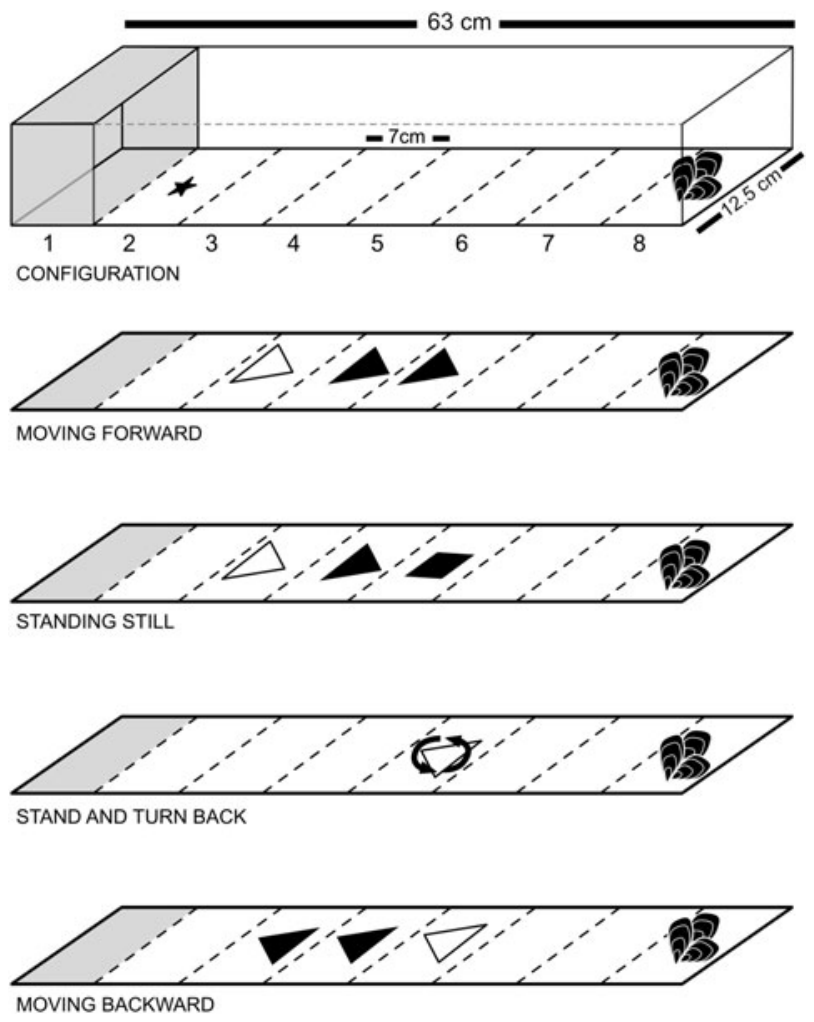

Fig. 1. Experimental setup and rules of behaviour used to simulate the correlated random walk and estimate the null distribution. The upper part presents the configuration. Eight positions separated by $7 \mathrm{~cm}$ are placed along a linear axis. A shelter is placed at the first position and a food source (crushed mussels) is placed at the last position. Two grids block the movements at both extremities of the experimental environment. The four lower parts present the possible movements, determined by the orientation of the individuals. They can move or stand, and stand still or turn back. Probabilities are determined by a sequence of binomial laws $\mathrm{B}(1,0.5)$ addressing each of the processes as a function of the previous one.
(Figure 1). A null model was derived from a correlated random walk (Renshaw \& Henderson, 1981; Reynolds, 2014). In this model, individuals explore space according to given probabilities to move, stand still or stand and turn (Figure 1). It is characterized as 'correlated' because unlike a simple random walk, probabilities in a correlated random walk are dependent on the individual's last direction of movement (i.e. a conditional probability). As a functional consequence, in a single coordinate axis, an individual cannot step back without having to turn back first. Probabilities are decomposed first, as an equal probability to move or stand, and second, as another equal probability to stand and maintain the same direction, or to stand and turn back. The realized distribution (specific to experimental condition) is considered biased from the simulated null distribution if probabilities defining movement change according to specific behaviours, the active search for food or shelter in our case (Farnsworth \& Beecham, 1999).

In this type of process, statistical distribution properties are therefore derived from the occupation probabilities (Renshaw \& Henderson, 1981); regarding experiments, observations consisted of recording, for each replicate $k=1 \ldots K$, the time spent $T_{k}(n)$ by an individual in each of the positions $n=$ $1 \ldots N$. The average is then calculated as:

$$
\bar{T}(n)=\frac{\sum_{k=1}^{K} T_{k}(n)}{K}
$$

which conserves the probability that:

$$
\sum_{n=1}^{N} \bar{T}(n)=T_{\exp }
$$

where $T_{\exp }$ is the total duration of the experiment.

Because of the lack of independence in data sets, and because it is very difficult to estimate $a$ priori biased probabilities for behavioural changes, a statistic $\delta$ was designed to compare two different experiences; it represents an integrated measure of the distance of each experimental outcome to the null distribution:

$$
\delta_{i}=\frac{N \sum_{n=1}^{N}\left(\bar{T}_{i}(n)-\bar{T}_{\mathrm{o}}(n)\right)^{2}}{T_{\exp , i}}
$$

with $T_{\exp , i}$ the duration of experiment $i$ (also used to calculate the free distribution), and $\bar{T}_{i}(n)$ and $\bar{T}_{\mathrm{o}}(n)$ the averages of time spent in the position $n$ for respectively, the experimental situation, $i$, and the calculated null distribution, o. The statistical test which compares two different experimental situations, $i$ and $j$ (i.e. 'strong' and 'dim' light conditions in our case) is then based on comparing the frequency distribution (or the probability law) of $\delta_{i}$ and $\delta_{j}$.

\section{Numerical simulation}

Numerical simulations are performed to complete two tasks, which are first to estimate the time spent in each of the positions in an experimental device and second to calculate the empirical frequency distribution of $\delta_{i}$ and $\delta_{j}$.

For the first task, the simulation is performed on the duration of an actual experiment. At each time step, the 
simulation determines at which position within the experimental environment an individual is. The sum of all time steps in each of the positions successively occupied over the entire duration of the experiment, allows calculation of $T_{i}(n)$. Physical frontiers (positions 1 and $N$ ) are closed for the organisms, so they can be considered as reflective barriers. Experiments are conceived as representing movements on a single coordinate axis. $\Delta x$ the space interval between 2 identified positions ( $\sim$ two times the size of the individuals) equals $L_{\text {max }} / N$, with $L_{\text {max }}$ the total length of the experimental raceway. The time step $\Delta t$, used for the simulation, is calculated as $\Delta x / v$, where $v$ is the average speed of the individuals. The initial position for the simulation is also the position at which individuals were placed at the beginning of the experiment (Figure 1). At each time (and in each position), a table of probability is applied, which was defined as following: it first determines if the organism moves $\left(p_{1}=0.5\right)$ or stands $\left(q_{1}=0.5\right)$, and then, in the second case, if it stands still $\left(p_{2}=0.5\right)$ or turns back $\left(q_{2}=0.5\right)$. The number of iterations for the simulation equals the number of replications in the experiments.

For the second task, the statistic $\delta_{i}$ was calculated with two sets of data, the first one corresponding to the numerical simulation of free distribution outcome and the second to the results of the experiment. To avoid having to define a model for a probability law that remains unknown, numerical simulation is used to construct empirical null distribution from the observed replicates. For this, a simple nonparametric bootstrap (Efron, 1981) is performed among $K$ replicates for $N-1$ positions: for each position $n$, one replicate $T_{k}(n)$ is withdrawn (with probability $1 / K$ ) with replacement, $K$ times, to reconstruct $K$ pseudoreplicates $T_{k}^{*}(n)$. One position is withdrawn randomly among all positions and is used to fulfil the condition:

$$
\sum_{n=1}^{N} T_{k}^{*}(n)=T_{\exp }
$$

which ensures the conservation of $T_{\text {exp }}$ for the calculation of the sum of the 'pseudo-average' (equation (2)).

$$
\sum_{n=1}^{N} \bar{T}^{*}(n)=T_{\exp }
$$

This was performed for both the null distribution and each of the experiments ('strong' and 'dim' light conditions), 500 times, in order to calculate a frequency distribution for $\delta_{i}^{*}$ and $\delta_{j}^{*}$ according to equation (3). The two empirical frequency distributions for $\delta_{i}^{*}$ and $\delta_{j}^{*}$ are compared by a Pearson $\chi^{2}$ test (Greenwood \& Nikulin, 1996), in which the frequency distribution corresponding to the distance between the 'dim' light experimental conditions and the null distribution simulation is used as the reference distribution.

The computer code has been written with Scilab 5.5.2, a free and open source software for modelling and analysis (distributed under CeCILL licence, GPL compatible, available at http://www.scilab.org).

\section{Experiments}

Experiments were carried out at the Sven Loven Center in Tjarnö, Sweden. Crabs used in the experiments were sampled in Tjärnö Bay, Hättebäcksvägen, Strömstad (Sweden), in early (6-9) April 2016, in early evening (18:00 - 20:30, local time). They were collected using crab traps with mussels as bait set at about $1 \mathrm{~m}$ water depth. Individuals were conditioned alone for $24 \mathrm{~h}$ in dark, to avoid acquiring a tolerance to light. For this, they were placed in cylindrical plastic containers (10 cm high and $8.5 \mathrm{~cm}$ diameter), with open water flow $\left(\mathrm{T}^{\circ} \mathrm{C}=6.5\right.$, salinity $\left.=30\right)$, and were not fed for this period.

Experiments were run after nightfall, when individuals of the two species were supposed to be at the peak of their circadian activity (McGaw \& Naylor, 1992; Lynch \& Rochette, 2007). The experimental setup consisted of a series of small raceways $(63 \mathrm{~cm} \mathrm{~L}$ and $12.5 \mathrm{~W}$ ) in which 8 sections of $7 \mathrm{~cm}$ each were delimited (Figure 1). The first position (1) was covered by a wood plank to create a shelter, and at the last position $(N)$ some potential food was placed $(30 \mathrm{~g}$ of crushed mussels). PVC grids, letting the water flow past but not the individuals, were placed at each end. The raceways were installed at the middle of large $(570 \mathrm{~cm} \mathrm{~L}, 108 \mathrm{~cm} \mathrm{~W}, 80 \mathrm{~cm}$ $\mathrm{H})$ outdoor fibreglass tanks and separated by wooden walls, allowing up to 12 replicate runs, simultaneously. During the experiment, the water height in the tanks was decreased to $8 \mathrm{~cm}$ and the flow (oriented from position $N$ to 1 , to potentially carry signals of the presence of food outward) was lowered to $201 \mathrm{~min}^{-1}$ in order to avoid horizontal speeds larger than $0.4 \mathrm{~cm} \mathrm{~s}^{-1}$, thus preventing the flow from reducing movements and foraging efficiency (Robinson et al., 2011). White lights were placed over the raceway to produce about $540 \mathrm{~lx}$ (between 520 and $560 \mathrm{~lx}$ at the water surface in all raceways), to represent 'strong' light conditions, while 'dim' light conditions were maintained at $3 \mathrm{~lx}$ (Franke et al., 2013).

Animals were handled gently and were not harmed during the experiments. All experiments were performed in a minimum of time respecting objectives and constraints. All animals were released at the place where they were caught initially at the end of the experiments.

\section{RESULTS}

The initial task was to determine the horizontal displacment speed of individuals. Individuals were placed in the experimental tank with the water flow used during experiments and their speeds were assessed by successive trials. An average of $0.35 \pm 0.08(\mathrm{SD}) \mathrm{cm} \mathrm{s}^{-1}$ was calculated from 33 individuals of the species Pagurus bernhardus and $0.20 \pm$ 0.06 (SD) $\mathrm{cm} \mathrm{s}^{-1}$ from 36 individuals of the species Carcinus maenas.

\section{Experiments with $P$. bernhardus}

Experiments were completed with the 33 individuals (used as replicates) for $1800 \mathrm{~s}$ each, and successively in dim and strong light conditions, for placing them in identical experimental conditions. Individuals were placed at the second position in a raceway, next to the sheltered position 'one' (Figure 1). Results of experiments for which individuals did not move at all from the initial position were removed from the data sets. This left 27 replicates for the 'dim' light condition and 32 replicates for the 'strong' light condition. Results are presented in Figure $2 \mathrm{~A}, \mathrm{C}$. The calculated value of $\chi_{\mathrm{obs}}^{2}=0.62$, measuring the distance between the frequency distributions 

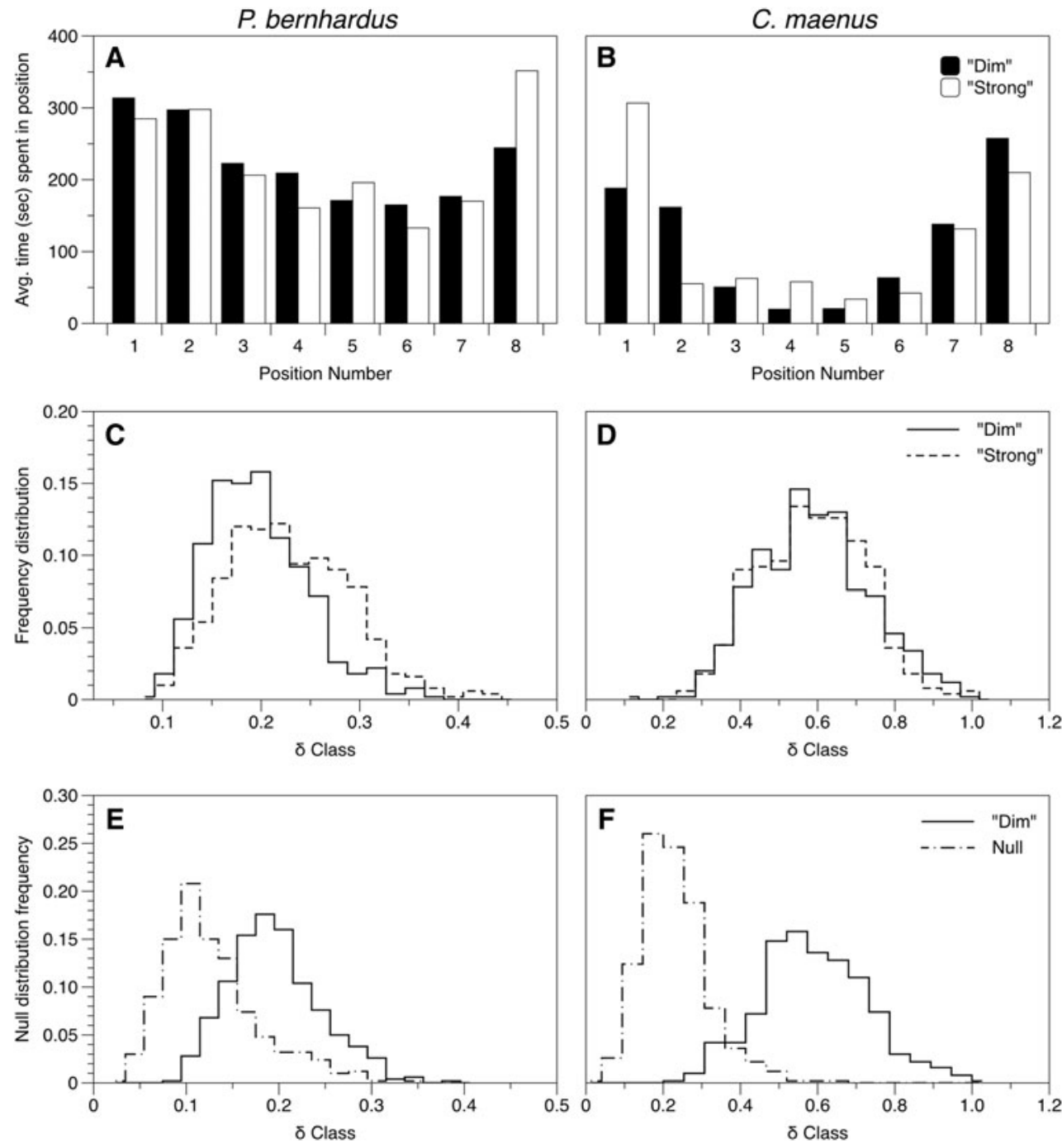

Fig. 2. Average time spent by Pagurus bernhardus (A) and by Carcinus maenas (B) individuals in each position of the experimental environment and the corresponding frequency distribution of the $\delta$ statistics (respectively $\mathrm{C}$ and $\mathrm{D}$ ) for both 'dim' and 'strong' light conditions experiments. The difference between the two frequency distributions was tested with a Pearson's $\chi^{2}$ homogeneity test. The test has shown that it is not possible to reject $\mathrm{H}_{0}$, suggesting an absence of significant differences. Frequency distributions of the $\delta$ statistics were calculated as both a deviation of the results of the 'dim' condition experiments from the null distribution and a deviation of the null distribution from itself, for both Pagurus bernhardus (E) and Carcinus maenas (F). Significant differences were assessed with the Pearson's $\chi^{2}$ homogeneity test, suggesting that the behaviours of individuals of both species are significantly different from a correlated random walk as we defined it.

of $\delta_{i}$ (corresponding to the dim condition) and $\delta_{j}$ (corresponding to the 'strong' light conditions), is not in the rejection area determined with the probability threshold of $\alpha=$ $0.05\left(\chi_{\text {obs }}^{2} Z=3.84\right)$. It is therefore impossible to reject $\mathrm{H}_{0}$, i.e. an absence of differences between the two distributions, for 'strong' and 'dim' light.

\section{Experiments with C. maenas}

Experiments were performed this time with only 25 individuals (used as replicates) for only $900 \mathrm{~s}$ each. Runs were done as above, with identical treatment, that is successively in 'dim' and 'strong' light conditions, and individuals were placed at the second position, next to the sheltered position 'one' (Figure 1). Results of the experiments for which individuals did not move at all from the initial position were removed from the data sets. Thus 22 replicates remained for the 'dim' light condition and 20 replicates for the 'strong' light condition. Results are presented in Figure $2 \mathrm{~B}, \mathrm{D}$. The calculated value of $\chi_{\text {obs }}^{2}=0.07$, measuring the distance between the frequency distributions of $\delta_{i}$ (corresponding to the 'dim' condition) and $\delta_{j}$ (corresponding to the 'strong' light conditions), is not in the rejection area determined with the probability threshold of $\alpha=0.05\left(\chi_{\text {obs }}^{2} Z=3.84\right)$. As for the hermit crab ( $P$. bernhardus), there is no significant difference $\left(\mathrm{H}_{\mathrm{o}}\right.$ cannot be rejected) between the two distributions, for 'dim' and 'strong' light.

\section{Testing the deviation from the null distribution}

To test if the results of the experiments performed in 'dim' light conditions deviate or not from the null distribution (correlated random walk), a Pearson $\chi^{2}$ test was done for distributions of $\delta_{i}$, corresponding to the 'dim' light conditions $(P$. bernhardus and C. maenas) and $\delta_{j}$, corresponding to deviation 
of the null distribution on itself (with parameters corresponding to the experiments for the two species respectively). Results are presented in Figure 2E, F. The test provided a calculated value of $\chi_{\mathrm{obs}}^{2}=4.70$ for $P$. bernhardus, and a calculated value of $\chi_{\text {obs }}^{2}=19.41$ for C. maenas, which are both in the rejection area determined with the probability threshold of $\alpha=0.05\left(\chi_{\text {obs }}^{2} Z=3.84\right)$. It is therefore possible to reject an absence of difference between the null and 'dim' light condition distributions.

\section{DISCUSSION}

There are several methods available for estimating null distributions. The choice between them can be difficult because there are links between distinctive classes of behaviour process simulation approaches (e.g. random-walk, Markov chains and stochastic process modelling). Besides, many variants (e.g. continuous $v s$ discrete) exist for each of the categories, each variant being developed to solve either mathematical or applied problems. Few studies actually compare different methods. In one of these rare studies, Gurarie et al. (2015) stated: 'Not surprisingly, the lack of a clear and agreed-upon framework for the selection of appropriate analysis tools has led to a wide array of approaches being applied to similar research questions. This methodological ambiguity has led to a crisis of choice where practitioners, particularly those new to the analysis of movement data, face difficulties deciding which tool to use for a given data set and question'. Founding articles of three important variants of these methods, continuous-time Markov (CTM) chains, correlated random walks and stochastic processes, date from the $1950 \mathrm{~s}$ and their applications to animal behavioural studies from the 1980s. The main publication on continuous-time Markov (CTM) models applied to behavioural studies was written by Metz et al. (1983), with few appearing since then. On the other hand, E. Renshaw and other authors have written many articles about correlated random walk and stochastic processes since 1981 (see Gurarie et al., 2015 for a methodological review), giving a significant advantage for the development of these methods to specific questions. In addition, a CTM Markov model would have been difficult to construct in our case. The 'states', as we perceive them, are clearly defined in two nested levels: the first one separating 'Moving Forward' and 'Not Moving Forward', and at the second level, when 'Not Moving forward', then 'Staying in the same direction' or 'Turning Back'. The transition probabilities between states (moving or stopping, and standing still or turning back) would be problematic to estimate from observations of successive positions in such a nested configuration. It would become even more complicated if we consider that, at the ends of the raceways, the probability to keep on moving in the same direction is zero. This cannot be considered as a reflective barrier per se because it increases the probability to stand still. Besides, in order to estimate transition probabilities, CT Markov models assume that the process is in a steady state at each instant, making parameters time-independent. Finally, in a CTM model, the distribution of time spent in each of the states is difficult to assess; it is particularly challenging to assume that this distribution is exponential (respectively, geometric for stochastic processes) for all states because of the nested structure of the state transition.
The alternative hypothesis $\left(\mathrm{H}_{1}\right)$ of this study implied that the exposure to a factor, recognized as having an effect on the activity of crab species, may modify the behaviour of individuals. However, it was found in the experimental data set that there were no significant differences between the results of the two conditions ('dim' light condition corresponding to the natural night-time environment and a 'strongly' contrasting light condition corresponding to a plausible disturbance), for either species: $P$. bernhardus (the hermit crab) or C. maenas (the green crab). These results suggest that the behaviours of these crab species were not, given the conditions of the experiments, affected by the light conditions, contrary to our expectation. However, what was considered as a 'dim' light here is difficult to compare to actual values available in the literature, because of our lack of knowledge about the spectral distribution of the light source, but all estimates suggest that crabs were placed in what has been characterized as highly light-polluted conditions (Kamrowski et al., 2012; Franke et al., 2013; Cinzano \& Falchi, 2014). These are reportedly similar to conditions encountered along urbanized coasts of North America and Europe (Cinzano et al., 2001).

Precautions were taken to minimize the changes in living conditions of the individuals used prior to experiments. Individuals were kept in dark and quiet conditions during the day before the tests, simulating sheltering. Water that flowed into the experimental tank was pumped directly from the bay, tanks were situated outdoors at ambient temperature, and the food source was identical to the one used to attract them to the traps initially. Experiments were carried out at night, respecting the circadian rhythm of these two species (Burrows et al., 1999; Turra \& Denadai, 2003). Nonetheless, other reasons, both experimental and ecological, may still explain the absence of any measured effect. The lack of temperature control may have led to an uncontrolled modification of the behaviour (Briffa et al., 2013), the absence of other environmental cues or the absence of contact with the food source for $24 \mathrm{~h}$ before experiments may have decreased their foraging efficiency (Tran, 2015), or an absence of light avoidance instinct, could explain the absence of a proximal effect of excess of light on the behaviour of the species tested.

In addition, the absence of difference between observations made in our experimental conditions does not signify that the distribution and the behaviours are random. Qualitatively, in all cases, individuals spent, on average, more time in the first and last positions than at the middle of the raceways, but this trend was far more pronounced for $C$. maenas than for $P$. bernhardus individuals (Figures $2 \mathrm{~B}, \mathrm{D}$ and $2 \mathrm{~A}, \mathrm{C}$ respectively). There were no inverse trends between experiments performed in 'dim' light conditions - when foraging activity was expected to predominate - and those performed under 'strong' light conditions - when sheltering behaviour was expected to predominate.

The null distribution may tend to a uniform distribution over a long term and increasing replicates, but no analytical calculation can demonstrate that this is the asymptotic property of our system dynamics. In other words, the simulated series, as the observed ones, cannot be considered as being ergodic. The null distribution is our reference state, which is here defined as a 'standard' state; it represents a situation for which no external factor influences, or changes, the behaviour from the 'standard' condition. Here, 'standard' conditions are where no behaviour predominates on the other, which 
implies that their probability to occur is uniformly distributed with alternatives at the same level of decision. It was assumed to occur when: (1) individuals are acclimated to the conditions and do not feel any stressing factors that can influence one behaviour rather than the other, and (2) individuals have a limited perception of their environment (a situation which implied that the experimental time had to remain short). However, under the most 'standard' conditions in which these experiments were performed (dim light), the behaviour cannot be assimilated to the correlated random walk we have simulated. The deviation from the null distribution is significant, suggesting that the experimental environment was constraining enough to trigger an oriented behaviour. The problem may lie in the identification of the table of probability used to describe the behaviour not being performed properly. Chapman (2000) pointed out that many experimental approaches tend to consider the set of possible animal behaviours in a manner which is oversimplified, often stating that it is invariant, neglecting local disturbances and overemphasizing the role of broad scale cues. The present experiment therefore suggests that the observed behaviour is not well understood and quantified under the apparently simple range of conditions tested. Here, in the raceway experiments, from a statistical point of view, the distribution of the time spent in each position along the track carried all information, but after analysing our results, it appears that future studies should record the displacement behaviour of the animals in more detail (in addition to recording the time in a particular position) to better define the tables of probabilities used to estimate the null distribution.

\section{CONCLUSIONS}

Bennie et al. (2014) summarized many possible direct or indirect effects of artificial light on animal behaviour and life cycles and there is a need to test proximal or direct effects. In the present case, the protocol for testing an effect was designed in a minimal way to be as straightforward as possible, but no effect was detected. The null distribution, which constitutes the only known reference, is, however different from the distribution observed in the 'dim' light conditions which is assumed to approximate conditions of absence of effect for all the organisms studied. If this statement is not true, if experimental conditions modify the behaviour, or if rapid-learning has already taken place, then this assumption would be invalid.

Our experiment raises more questions than it answered, but it constitutes an invitation to increase our knowledge of the behaviour of the studied organisms, which is ultimately a prerequisite to all studies performed on the ecology of organisms. The central problem of our study was to define a well-described reference state to which a comparison can be made based on a reasonable conception of the animal behaviour in the experimental device. As such we compare our results not to any notion of natural or laboratory conditions (Underwood, 2009), but to a limited set of behaviours that can be described by the model. Our contribution has been to tackle this problem by recreating a null distribution from an ad hoc completely random behaviour. However, the corresponding correlated random walk model is based on concepts that do not rest on knowledge about the behaviour of an individual crab, but rather on common principles, and the resulting distribution appeared to be far from the distribution observed in 'dim' light conditions. Still important information about the motion and impetus are missing from these experiments. The prerequisite to have a well-established, accurate description of individual behaviours (prior to any test on the potential effect of disturbance factors) however rarely exists in behavioural ecology, which does not rest on any kind of fundamental scientific laws. Animal behaviour is not a fixed property of species; there is ample information describing both positive and negative responses of species to increasing urbanization, for example (Sol et al., 2013). For our particular case, an increase of knowledge can be nonetheless be achieved in the future, using more sophisticated sensors (e.g. motion sensors and accelerometers) and imagery-based approaches to produce a more complete description of animal behaviours.

\section{ACKNDWLEDGEMENTS}

The authors are very grateful to Luz Amadei Martínez, María del Carmen Blanco Fernández, Amanda Goble, Susann Lüneburg, Shraveena Venkatesh and Robin Weber for performing the experiments and sharing the data. This work was supported by the International Master in Marine Biodiversity and Conservation (EMBC+). The authors would like to thank also the Sven Loven Marine Center of the Götenborg University and all its dedicated staff for having hosted them and allowed this study to be conducted.

\section{FINANCIAL SUPPDRT}

The work of J. Coston-Guarini was supported by the 'Laboratoire d'Excellence' LabexMER (ANR-10-LABX-19) and co-funded by a grant from the French government under the program 'Investissements d'Avenir'.

\section{REFERENCES}

Bennie J., Davies T.W., Inger R. and Gaston K.J. (2014) Mapping artificial lightscapes for ecological studies. Methods in Ecology and Evolution 5, 534-540.

Brady J. (2013) Behavioral rhythms in invertebrates. In Achoff J. (ed.) Biological rhythms, Volume 4. New York, NY: Springer-Verlag, pp. 125-144.

Briffa M., Bridger D. and Biro P.A. (2013) How does temperature affect behaviour? Multilevel analysis of plasticity, personality and predictability in hermit crabs. Animal Behaviour 86, 47-54.

Brüning A., Hölker F., Franke S., Preuer T. and Kloas W. (2015) Spotlight on fish: light pollution affects circadian rhythms of European perch but does not cause stress. Science of the Total Environment 511, 516-522.

Burrows M.T., Kawai K. and Hughes R.N. (1999) Foraging by mobile predators on a rocky shore: underwater TV observations of movements of blennies Lipophrys pholis and crabs Carcinus maenas. Marine Ecology Progress Series 187, 237-250.

Campbell D.L.M., Weiner S.A., Starks P.T. and Hauber M.E. (2009) Context and control: behavioural ecology experiments in the laboratory. Annales Zoologici Fennici 46, 112-123. 
Carlton J.T. and Cohen A.N. (2003) Episodic global dispersal in shallow water marine organisms: the case history of the European shore crabs Carcinus maenas and C. aestuarii. Journal of Biogeography 30, 18091820 .

Chakravarti L.J. and Cotton P.A. (2014) The effects of a competitor on the foraging behaviour of the shore crab Carcinus maenas. PLoS ONE 9, e93546. doi: 10.1371/journal.pone.0093546.

Chapman M.G. (2000) Poor design of behavioural experiments gets poor results: examples from intertidal habitats. Journal of Experimental Marine Biology and Ecology 250, 77-95.

Cinzano P. and Falchi F. (2014) Quantifying light pollution. Journal of Quantitative Spectroscopy and Radiative Transfer 139, 13-20.

Cinzano P., Falchi F. and Elvidge C.D. (2001) The first world atlas of the artificial night sky brightness. Monthly Notices of the Royal Astronomical Society 328, 689-707.

Cohen A.N. and Carlton J.T. (1995) Non-indigenous aquatic species in a United States estuary: a case study of the biological invasions of the San Francisco Bay and Delta. Biological Studies. Washington, DC: U.S. Fish and Wildlife Service.

Davies N.B., Krebs J.R. and West S.A. (2012) An introduction to behavioural ecology, 4th edition. Oxford: Wiley-Blackwell.

Efron B. (1981) Nonparametric estimates of standard error: the jackknife, the bootstrap and other methods. Biometrika 68, 589-599.

Farnsworth K.D. and Beecham J.A. (1999) How do grazers achieve their distribution? A continuum of models from random diffusion to the ideal free distribution using biased random walks. American Naturalist 153, 509-526.

Franke S., Brüning A., Hölker F. and Kloas W. (2013) Study of biological action of light on fish. Journal of Light and Visual Environment 37 $1-11$.

Greenwood P.E. and Nikulin M.S. (1996) A guide to chi-squared testing Applied probability and statistics. New York, NY: John Wiley and Sons.

Gurarie E., Bracis C., Delgado M., Meckley T.D., Kojola I. and Wagner M. (2015) What is the animal doing? Tools for exploring behavioural structure in animal movements. Journal of Animal Ecology 85, 69-84.

Kamrowski R.L., Limpus C., Moloney J. and Hamman M. (2012) Coastal light pollution and marine turtles: assessing the magnitude of the problem. Endangered Species Research 19, 85-98.

Lynch B.R. and Rochette R. (2007) Circatidal rhythm of free-roaming sub-tidal green crabs, Carcinus maenas, revealed by radio-acoustic positional telemetry. Crustaceana 80, 345-355.

Martin P. and Bateson P. (2007) Measuring behaviour: an introductory guide, 3rd edition. Cambridge: Cambridge University Press.

McGaw I.J. and Naylor E. (1992) Distribution and rhythmic locomotor patterns of estuarine and open-shore populations of Carcinus maenas. Journal of the Marine Biological Association of the United Kingdom 72, 599-609.

McGaw I.J. and Penney C.M. (2014) Effect of meal type on specific dynamic action in the green crab, Carcinus maenas. Journal of Comparative Physiology B 184, 425-436.

Metz H.A.J., Dienkse H., de Jonge G. and Putters F.A. (1983) Continuous-time Markov chains as models for animal behaviour. Bulletin of Mathematical Biology 45, 643-658.
Moore M.V., Pierce S.M., Walsh H.M., Kvalvik S.K. and Lim J.D. (200o) Urban light pollution alters the diel vertical migration of Daphnia. Verhandlungen des Internationalen Verein Limnologie 27, 1-4.

Navarro-Barranco C. and Hughes L.E. (2015) Effects of light pollution on the emergent fauna of shallow marine ecosystems: amphipods as a case study. Marine Pollution Bulletin 94, 235-240.

Nickell T. D. and Moore P.G. (1992) The behavioural ecology of epibenthic scavenging invertebrates in the Clyde Sea area: laboratory experiments on attractions to bait in moving water, underwater TV observations in situ and general conclusions. Journal of Experimental Marine Biology and Ecology 159, 15-35.

Pihl L. (1985) Food selection and consumption of mobile epibenthic fauna in shallow marine areas. Marine Ecology Progress Series 22, 169-179.

Poulin C., Bruyant F., Laprise M.-H., Cockshutt A.M., Vandenhecke J.M.-R. and Huot Y. (2013) The impact of light pollution on diel changes in the photophysiology of Microcystis aeruginosa. Journal of Plankton Research 36, 286-291.

Quinn B.K., Boudreau M.R. and Hamilton D.J. (2012) Inter- and intraspecific interactions among green crabs (Carcinus maenas) and whelks (Nucella lapillus) foraging on blue mussels (Mytilus edulis). Journal of Experimental Marine Biology and Ecology 412, 117-125.

Ramsay K., Kaiser M.J. and Hughes R.N. (1997) A field study of intraspecific competition for food in hermit crabs (Pagurus bernhardus). Estuarine, Coastal and Shelf Science 44, 213-220.

Renshaw E. and Henderson R. (1981) The correlated random walk Journal of Applied Probability 18, 403-414.

Reynolds A.M. (2014) Towards a mechanistic framework that explains correlated random walk behaviour: correlated random walkers can optimize their fitness when foraging under the risk of predation. Ecological Complexity 19, 18-22.

Robinson E.M., Smee D.L. and Trussell G.C. (2011) Green crab (Carcinus maenas) foraging efficiency reduced by fast flows. PLoS ONE 6, e21025. doi: 10.1371/journal.pone.0021025.

Sol D., Lapiedra O. and González-Lagos C. (2013) Behavioural adjustments for a life in the city. Animal Behaviour 85, 1101-1112.

Tran M.V. (2015) Behavioral reactions to novel food odors by intertidal hermit crabs. Behavioral Processes 113, 35-40.

Turra A. and Denadai M.R. (2003) Daily activity of four tropical intertidal hermit crabs from southeastern Brazil. Brazilian Journal of Biology 63, 537-544

Underwood A.J. (2009) Components of design in ecological field experiments. Annales Zoologici Fennici 46, 93-111.

Vickers M. and Schwarzkopf L. (2016) A random walk in the park: an individual-based model for behavioral thermoregulation. American Naturalist $187,481-490$.

and

Ylonen H. and Wolff J.O. (1999) Experiments in behavioural ecology and the real world. Trends in Ecology and Evolution 14, 82.

\section{Correspondence should be addressed to:}

J.-M. Guarini

The Entangled Bank Laboratory,

66650 Banyuls sur Mer, France

email: jm.guarini@entangled-bank-lab.org 